\title{
Magnetic Resonance Imaging Followup of Temporomandibular Joint Inflammation, Deformation, and Mandibular Growth in Juvenile Idiopathic Arthritis Patients Receiving Systemic Treatment
}

\author{
Andrea Bollhalder, Raphael Patcas (D), Martina Eichenberger, Lukas Müller, \\ Silke Schroeder-Kohler, Rotraud Katharina Saurenmann iD, and \\ Christian Johannes Kellenberger (iD)
}

\begin{abstract}
Objective. To investigate the course of temporomandibular joint (TMJ) inflammation, osseous deformation, and mandibular ramus growth in children with juvenile idiopathic arthritis (JIA) during systemic therapy.

Methods. Longitudinal study of 38 consecutive patients with JIA (29 female, median age 9.0 yrs, interquartile range 6.2-10.7 yrs) receiving systemic therapy with TMJ involvement, with 2 TMJ magnetic resonance imaging (MRI) examinations $\geq 2$ years apart and no TMJ corticosteroid injection. Clinical and MRI findings were compared between initial and followup examinations and between TMJ with and without active inflammation at baseline.

Results. Over a median period of 3.6 years (range, 2.0-8.7 yrs), MRI grade of TMJ inflammation improved $(p=0.009)$ and overall osseous deformity tended to become less severe $(p=0.114)$. In TMJ with arthritis at baseline $(46 \mathrm{TMJ})$, both the grades of inflammation $(\mathrm{p}<0.001)$ and deformity $(\mathrm{p}=0.011)$ improved. In TMJ with no arthritis at baseline (30 TMJ), the frequency and grade of condylar deformation remained stable. Mandibular ramus growth rates were not significantly different between TMJ with and without arthritis at baseline $(1.3 \mathrm{~mm} / \mathrm{yr}$ vs $1.5 \mathrm{~mm} / \mathrm{yr}, \mathrm{p}=0.273)$, and were not correlated with the degree of inflammation at baseline or followup. The frequency of facial asymmetry tended to be lower at followup than at initial examination $(24 \%$ vs $45 \%, p=0.056)$.

Conclusion. Our results suggest that systemic treatment of TMJ arthritis in children with JIA decreases the degree of inflammation seen on MRI, preserves osseous TMJ morphology, and maintains normal mandibular ramus growth. (First Release December 15 2019; J Rheumatol 2020;47:909-16; doi:10.3899/jrheum.190168)
\end{abstract}

Key Indexing Terms:

TEMPOROMANDIBULAR JOINT

MAGNETIC RESONANCE IMAGING

\section{JUVENILE IDIOPATHIC ARTHRITIS OUTCOME RESEARCH}

Involvement of the temporomandibular joint (TMJ) is common in patients with juvenile idiopathic arthritis (JIA), with an estimated frequency of about $40-60 \%$ based on large imaging series in the literature ${ }^{1,2}$. As inflammation of the TMJ is suspected to be the cause for craniofacial growth disturbances frequently seen in patients with $\mathrm{JIA}^{3}$, early detection and prompt treatment of arthritis is currently thought to be essential for normal development of the TMJ and mandible in growing children.

Because TMJ arthritis may often be asymptomatic and
From the Department of Diagnostic Imaging, the Division of Paediatric Rheumatology, and the Children's Research Center, University Children's Hospital Zürich; Clinic of Orthodontics and Paediatric Dentistry, Center of Dental Medicine, University of Zürich, Zürich; Department of Child and Adolescent Medicine, Kantonsspital Winterthur, Winterthur, Switzerland.

A. Bollhalder, DDS, Orthodonist, Department of Diagnostic Imaging, University Children's Hospital Zürich, and Clinic of Orthodontics and Paediatric Dentistry, Center of Dental Medicine, University of Zürich; R. Patcas, DDS, PhD, PD, Orthodonist, Clinic of Orthodontics and Paediatric Dentistry, Center of Dental Medicine, University of Zürich; $M$. Eichenberger, DDS, Orthodontist, Clinic of Orthodontics and Paediatric Dentistry, Center of Dental Medicine, University of Zürich; L. Müller, DDS, Orthodontist, Clinic of Orthodontics and Paediatric

Dentistry, Center of Dental Medicine, University of Zürich;

S. Schroeder-Kohler, MD, Pediatric Rheumatologist, Division of
Paediatric Rheumatology, University Children's Hospital Zürich, and Children's Research Center, University Children's Hospital Zürich; R.K. Saurenmann, MD, Professor, Pediatric Rheumatologist, Director, Department of Child and Adolescent Medicine, Kantonsspital Winterthur, and Division of Paediatric Rheumatology, University Children's Hospital Zürich, and Children's Research Center, University Children's Hospital Zürich; C.J. Kellenberger, MD, Professor, Pediatric Radiologist, Radiologist-in-Chief, Department of Diagnostic Imaging, and Children's Research Center, University Children's Hospital Zürich.

Address correspondence to Dr. C.J. Kellenberger, Department of Diagnostic Imaging, University Children's Hospital Zürich, Steinwiesstrasse 75, CH 8032 Zürich, Switzerland.

E-mail: Christian.Kellenberger@kispi.uzh.ch Accepted for publication September 3, 2019. 
difficult to diagnose clinically ${ }^{4,5}$, contrast-enhanced magnetic resonance imaging (MRI) is considered the best available method for early diagnosis ${ }^{6}$. In addition, MRI allows for grading the level of inflammation in the TMJ as well as assessment of the osteochondral joint morphology and height of the mandibular ramus ${ }^{7,8,9}$.

Our group has previously shown that intraarticular corticosteroid injections in a cohort of 33 children with JIA neither preserved normal growth of the mandibular ramus over a median period of 5 years, nor prevented progressive TMJ deformity ${ }^{10}$.

With this study, we aimed to evaluate mandibular growth, the course of inflammation, and deformity of the TMJ in children who underwent systemic immunosuppressive treatment for JIA.

\section{MATERIALS AND METHODS}

Patients. For this retrospective study, we identified 38 consecutive children seen at our tertiary pediatric university hospital between 2006 and 2015 with a diagnosis of JIA according to the International League of Associations for Rheumatology 2001 criteria $^{11}$, MRI diagnosis of TMJ involvement (TMJ arthritis and/or TMJ deformity presumed to be the result of arthritis), and an MRI followup after 2 or more years. During this period, we saw 479 patients with JIA in our outpatient clinics and MRI was performed routinely at a timepoint when TMJ involvement had a potential implication for treatment ${ }^{12}$. Patients without systemic immunosuppressive treatment and those who received any corticosteroid injections in the TMJ were excluded from the study. Children with no consent for retrospective data analysis were not considered. The study was conducted according to Swiss legislation and approved by the governmental research ethics committee (KEK ZH 2015-0433).

Patient data, including results of clinical examinations and medication during the observation period, were retrieved from the electronic patient files. The study population consisted of 29 girls and 9 boys, with a median age of 9.0 years at first MRI [age range 1.5-13.7 yrs, interquartile range (IQR) 6.2-10.7 yrs] and a median age of 6.8 years at initial diagnosis of JIA (age range 1.2-12.8 yrs, IQR 3.3-9.0 yrs). The followup MRI evaluated for this study was performed after a median interval of 3.6 years (range 2.0-8.7 yrs, IQR 2.6-4.7 yrs). The characteristics of the study population are summarized in Table 1. The terminology used in this work adheres to the recommendations by the TMJ Juvenile Arthritis Working Group ${ }^{13}$.

Clinical examination. Clinical assessment of the TMJ was performed as routine care at regular intervals by experienced pediatric rheumatologists and orthodontists ${ }^{14}$. Results of the examination closest to the respective MRI were used for this study (median interval between clinical assessment and MRI 0 days, IQR -2.2 to $0.8 \mathrm{mos}$ ). Presence of TMJ pain as reported by the patient and on palpation in a relaxed position of the mandible and during movement was noted. Mandibular skeletal asymmetry was graded as absent $(0)$, mild $( \pm 1)$, or severe $( \pm 2)$ with a deviation to the right (positive value) or to the left (negative value). Maximal mouth opening capacity (MOC; i.e., the unassisted greatest interincisal distance without adjustment for overbite) was measured with an acrylic ruler after the patient opened the mouth as wide as possible several times for warmup. Centiles of MOC were calculated from normal age- and sex-adjusted values ${ }^{15}$.

MRI evaluation. All contrast-enhanced MRI of the TMJ was performed at 1.5 Tesla (Signa MR/i Twinspeed or Discovery MR450, GE Medical Systems) with a TMJ surface coil in closed-mouth position according to the institutional protocol ${ }^{10}$. The MRI studies were reviewed by an orthodontist (AB) and a pediatric radiologist (CJK) in a consensus reading. As described previously ${ }^{8,10}$, TMJ involvement was graded with a progressive scoring system (Appendix, available from the authors on request). Presence and
Table 1. Characteristics of 38 children with JIA receiving systemic therapy and having TMJ involvement.

\begin{tabular}{lc}
\hline Characteristics & Values \\
\hline Female & $29(76)$ \\
Oligoarticular & $7(18)$ \\
Oligoarticular extended & $5(13)$ \\
Polyarticular RF-negative & $17(45)$ \\
Enthesitis-related arthritis & $2(5)$ \\
Psoriatic arthritis & $2(5)$ \\
Systemic arthritis & $1(3)$ \\
Unclassified arthritis & $4(11)$ \\
Age at diagnosis, yrs, median (IQR) & $6.8(3.3-9.0)$ \\
Age at initiation of systemic medication, yrs, & \\
$\quad$ median (IQR) & $8.1(4.8-9.9)$ \\
Age at first MRI, yrs, median (IQR) & $9.0(6.2-10.7)$ \\
Disease duration at first MRI, yrs, median (IQR) & $1.2(0.3-2.7)$ \\
MRI followup, yrs, median (IQR) & $3.6(2.6-4.7)$ \\
Duration of systemic medication between MRI & \\
$\quad$ studies, yrs, median (IQR) & $3.0(1.9-4.2)$ \\
\hline
\end{tabular}

Data are $\mathrm{n}(\%)$ unless otherwise indicated. JIA: juvenile idiopathic arthritis; TMJ: temporomandibular joint; RF: rheumatoid factor; IQR: interquartile range; MRI: magnetic resonance imaging.

degree of joint effusion, synovial thickening, and bone marrow edema were assessed on fat-saturated T2-weighted images. Presence and extension of joint enhancement was assessed on early contrast-enhanced images. Inflammatory activity of the TMJ was graded semiquantitatively on a 5 -grade scale (grades 0-4). Shape and integrity of the temporal bone (articular eminence and glenoid fossa) and mandibular condyle were assessed on gradient echo images. Osseous deformity was also graded semiquantitatively on a 5-grade scale. Mandibular ramus height was measured on minimum intensity projection images from a 3-D gradient echo sequence, on a line parallel to the posterior border of the ramus through the most cranial point of the condyle to the intersection with the inferior border of the ramus ${ }^{9}$. From the mandibular height at the initial MRI and followup MRI, growth rates were calculated for each mandibular ramus, and compared to normal age- and sex-matched growth rates based on longitudinal cephalographic measurements between the condylion and gonion in 102 children from 3 to 16 years of age ${ }^{16,17}$.

Comparisons and statistical analysis. Descriptive data are given as mean $\pm \mathrm{SD}$ for continuous variables with normal distribution and as median (IQR) for variables without normal distribution. Normal distribution of the data was checked with the Shapiro-Wilk test. Frequencies are reported as fractions (percentage).

The clinical findings were compared between initial and followup examination with the chi-square test for frequencies, Wilcoxon test for ordinal and not normally distributed data, and paired sample $t$ test for data with normal distribution. TMJ pain, MOC, and facial asymmetry were correlated to MRI findings with the Spearman rank correlation.

The MRI findings were compared between initial and followup studies for TMJ with and without active inflammation at baseline, and between TMJ with and without active inflammation at baseline.

Frequencies of MRI findings at initial and followup examinations were compared with the chi-square test. Mandibular ramus height, grades of inflammation, and grades of deformity were compared with the Wilcoxon test between MRI examinations. Mandibular ramus growth rates were compared between TMJ with and without active inflammation at baseline with the Mann-Whitney U test. Measured growth rates were compared to expected normal growth rates with the Wilcoxon test. Growth rates were correlated with the grades of inflammation and deformity at initial and followup MRI using Spearman rank correlation. All statistical analyses were

Personal non-commercial use only. The Journal of Rheumatology Copyright @ $\odot 2020$. All rights reserved 
performed with MedCalc Statistical Software version 18.11.3 (MedCalc Software). A p value $<0.05$ was considered significant.

\section{RESULTS}

Medication. The indication for systemic immunosuppressive therapy was arthritis of multiple joints $(n=30$, peripheral joints and TMJ), arthritis of the spine or sacroiliac joints $(n=5)$, or uveitis $(n=2)$. In only 1 patient, severe TMJ arthritis was the main indication for systemic treatment, at a time when we no longer offered intraarticular corticosteroid injections to the TMJ. Before 2013, we would have considered corticosteroid injection in the case of isolated TMJ arthritis or when peripheral arthritis was under control by local therapy.

In $19 / 38$ children $(50 \%)$ the systemic medication was started 2.3 years (IQR 4.4-0.9 yrs) before the first MRI study. In the other half of the children, systemic medication was introduced at or after first MRI, with a median interval of 0.5 years (IQR 0.1-1.6 yrs). The median duration of systemic therapy between the initial and followup MRI was 3.0 years (IQR 1.9-4.2 yrs), corresponding to 96\% (IQR 72-100\%) of the observation period.

Systemic medication included methotrexate (MTX) in $35 / 38$ patients $(92 \%)$. During the observation period, MTX was substituted by another drug in 19/38 patients (50\%): leflunomide in 9 cases, etanercept (ETN) in 6 cases, infliximab in 2 cases, and golimumab (GOL) in 2 cases. MTX or leflunomide was combined with another drug in 20/38 patients (53\%): GOL in 9 cases, ETN in 9 cases, or tocilizumab in 2 cases. Two patients were treated with hydroxychloroquine and 1 patient with a combination of systemic corticosteroids, azathioprine, and GOL.

Clinical findings. The clinical findings at the initial and followup examinations are summarized in Table 2 . Tenderness was reported in different TMJ at baseline and at followup, with low frequencies of $13 \%$ and $9 \%$, respectively. Presence of TMJ pain did not correlate with MRI grades of

Table 2. Clinical findings in 38 patients with JIA receiving systemic therapy and having TMJ involvement.

\begin{tabular}{lccc}
\hline Variables & $\begin{array}{c}\text { Initial } \\
\text { Examination }\end{array}$ & $\begin{array}{c}\text { Followup } \\
\text { Examination }\end{array}$ & $\mathrm{p}$ \\
\hline Tenderness (76 TMJ) & $10 / 76(13)$ & $7 / 76(9)$ & $0.432^{*}$ \\
Asymmetry & $17 / 38(45)$ & $9 / 38(24)$ & $0.056^{*}$ \\
$\quad$ Frequency & & & $0.173^{* *}$ \\
$\quad$ Grade & $21 / 38(55)$ & $29 / 38(76)$ & \\
$\quad$ None, 0 & $16 / 38(42)$ & $6 / 38(16)$ & \\
$\quad$ Mild, $-1,1$ & $1 / 38(3)$ & $3 / 38(8)$ & \\
$\quad$ Severe, $-2,2$ & $44 \pm 8 \mathrm{~mm}$ & $49 \pm 8 \mathrm{~mm}$ & $<0.001^{* * *}$ \\
MOC, mm (mean $\pm \mathrm{SD})$ & $51 \pm 33$ & $53 \pm 29$ & $0.680^{* * *}$ \\
MOC, centiles (mean $\pm \mathrm{SD})$ & $51 \pm 3$ \\
\hline
\end{tabular}

Values are $\mathrm{n} / \mathrm{N}(\%)$ unless otherwise specified. Comparison between initial and followup examination with $*$ chi-square test, $* *$ Wilcoxon test, *** paired samples $t$ test. JIA: juvenile idiopathic arthritis; TMJ: temporomandibular joint; MOC: mouth opening capacity. inflammation or deformity. Mandibular skeletal asymmetry was noted in 17/38 patients (45\%) initially and in $9 / 38$ patients (24\%) at followup, with resolution of mild asymmetry in 10 patients, but development of mild asymmetry and deterioration of mild to severe asymmetry in 2 patients each. Presence and degree of asymmetry were not significantly different between initial and followup examination, but the frequency of asymmetry tended to be lower at followup (17/38 initially, vs 9/38 at followup, $\mathrm{p}=0.056$; Supplementary Figure 1, available from the authors on request). The degree of asymmetry showed a weak correlation with the grade of TMJ deformity $\left(r_{s}=0.316\right.$, $\mathrm{p}=0.005)$. The mean MOC improved by $4.4 \mathrm{~mm}(95 \% \mathrm{CI}$ 2.7-6.2 mm, p < 0.0001), but age- and sex-adjusted centiles of MOC were not significantly different (mean difference 1.6, 95\% CI -6.4 to 9.7, $\mathrm{p}=0.680$ ) between initial and followup assessment. Both absolute values and centiles of MOC showed no correlation with MRI grades of inflammation or deformity.

MRI findings. The prevalence of TMJ arthritis (active inflammation) was $27 / 38$ patients $(71 \%)$ at baseline and $23 / 38$ patients (61\%) at followup. TMJ deformity was seen in 22/38 patients $(59 \%)$ initially and in $21 / 38$ patients $(55 \%)$ at followup. Initially $7 / 38$ patients $(18 \%)$ had unilateral and $15 / 38$ (39\%) bilateral deformity. During the study period, 4/38 patients $(11 \%)$ developed unilateral and 1/38 (3\%) bilateral deformity, while in 4/38 (11\%) unilateral deformity and in 1/38 (3\%) bilateral deformity resolved. In another 1/38 patients $(3 \%)$, bilateral deformity improved to unilateral deformity. Therefore the overall frequency of unilateral and bilateral deformity was not significantly different at followup: $7 / 38$ patients (18\%) had unilateral and 14/38 (37\%) bilateral TMJ deformity.

At initial MRI, 46/76 TMJ (61\%) showed signs of inflammation (grade $>0$ ) and 37/76 (49\%) TMJ had some deformity (grade $>0$ ). At followup MRI, 40/76 TMJ (54\%) showed signs of inflammation and 35/76 TMJ (46\%) were deformed. Overall, grades of inflammation improved $(p=0.009)$, while grades of deformation were not significantly different $(\mathrm{p}=0.114)$ at followup. The inflammatory and deformity grades at the initial and followup MRI are detailed in Table 3 for all TMJ and groups of TMJ with and without inflammation at baseline. No TMJ showed the most severe grade of inflammation (inflammatory activity grade 4, TMJ filled with and expanded by pannus), large erosions, fragmentation of the condyle, or intraarticular calcification (deformity grade 4).

In the TMJ without inflammation at baseline, signs of inflammation were seen at followup in 10/30 TMJ (33\%), while the frequency and grades of deformation were not significantly different between initial and followup MRI.

In the TMJ with inflammation at baseline, the frequency and grades of inflammation improved significantly $(\mathrm{p}<0.001)$ at followup, as did the grades of deformity $(\mathrm{p}=0.011)$. 
Table 3. MRI findings in 38 patients with JIA receiving systemic therapy and having TMJ involvement.

\begin{tabular}{|c|c|c|c|}
\hline & Initial MRI & Followup MRI & $\mathrm{p}$ \\
\hline \multicolumn{4}{|c|}{ All TMJ, $n=76$} \\
\hline \multicolumn{4}{|c|}{ Inflammation } \\
\hline Frequency & $46 / 76(60.5)$ & 40/76 (53.6) & $0.392 *$ \\
\hline Grade & & & $0.009 * *$ \\
\hline 0 & $30 / 76(39.5)$ & 36/76 (47.4) & \\
\hline 1 & $28 / 76(36.8)$ & $36 / 76(47.4)$ & \\
\hline 2 & $14 / 76(18.4)$ & $4 / 76(5.3)$ & \\
\hline 3 & $4 / 76(5.3)$ & & \\
\hline \multicolumn{4}{|l|}{ Deformation } \\
\hline Frequency & $37 / 76(48.7)$ & $35 / 76(46.1)$ & $0.749 *$ \\
\hline Grade & & & $0.114 * *$ \\
\hline 0 & $39 / 76(51.3)$ & 41/76 (53.9) & \\
\hline 1 & $23 / 76(30.3)$ & $27 / 76(35.5)$ & \\
\hline 2 & $9 / 76(11.8)$ & $5 / 76(6.6)$ & \\
\hline 3 & $5 / 76(6.6)$ & $3 / 76(3.9)$ & \\
\hline \multicolumn{4}{|c|}{ TMJ without inflammation at baseline, $\mathrm{n}=30$} \\
\hline \multicolumn{4}{|c|}{ Inflammation } \\
\hline Frequency & 0/30 (0) & $10 / 30(33.3)$ & $<0.001 *$ \\
\hline Grade & & & $0.002 * *$ \\
\hline 0 & $30 / 30(100)$ & $20 / 30(66.7)$ & \\
\hline 1 & & $8 / 30(26.7)$ & \\
\hline 2 & & $2 / 30(6.7)$ & \\
\hline \multicolumn{4}{|l|}{ Deformation } \\
\hline Frequency & $13 / 30(43.3)$ & $17 / 30(56.7)$ & $0.303 *$ \\
\hline \multicolumn{4}{|l|}{ Grade } \\
\hline 0 & $17 / 30(56.7)$ & $13 / 30(43.3)$ & $0.375 * *$ \\
\hline 1 & $8 / 30(26.7)$ & $13 / 30(43.3)$ & \\
\hline 2 & $4 / 30(13.3)$ & $3 / 30(10.0)$ & \\
\hline 3 & $1 / 30(3.3)$ & $1 / 30(3.3)$ & \\
\hline \multicolumn{4}{|c|}{ TMJ with inflammation at baseline, $\mathrm{n}=46$} \\
\hline \multicolumn{4}{|c|}{ Inflammation } \\
\hline Frequency & $46 / 46(100)$ & $30 / 46(65.2)$ & $<0.001 *$ \\
\hline \multicolumn{4}{|l|}{ Grade } \\
\hline 0 & $0 / 46(0)$ & $16 / 46(34.8)$ & $<0.001 * *$ \\
\hline 1 & $28 / 46(60.9)$ & $28 / 46(60.9)$ & \\
\hline 2 & $14 / 46(30.4)$ & $2 / 46(4.3)$ & \\
\hline 3 & $4 / 46(8.7)$ & & \\
\hline \multicolumn{4}{|l|}{ Deformation } \\
\hline Frequency & $24 / 46(52.2)$ & 18/46 (39.1) & $0.210 *$ \\
\hline \multicolumn{4}{|l|}{ Grade } \\
\hline 0 & $22 / 46(47.8)$ & $28 / 46(60.9)$ & $0.011 * *$ \\
\hline 1 & $15 / 46(32.6)$ & $15 / 46(30.4)$ & \\
\hline 2 & $5 / 46(10.9)$ & $2 / 46(4.3)$ & \\
\hline 3 & $4 / 46(8.7)$ & $2 / 46(4.3)$ & \\
\hline
\end{tabular}

Values are $\mathrm{n} / \mathrm{N}(\%)$ unless otherwise specified. Comparison between MRI studies with $*$ chi-square test, ** Wilcoxon test. TMJ: temporomandibular joint; JIA: juvenile idiopathic arthritis; MRI: magnetic resonance imaging.

Inflammatory grades at followup MRI were lower for TMJ without inflammation at baseline than for TMJ with inflammation at baseline (Mann-Whitney U test, $\mathrm{p}=0.018$ ). Deformation grades were not significantly different between TMJ with and without inflammation at baseline both at initial (Mann-Whitney U test, $\mathrm{p}=0.454$ ) and followup MRI (Mann-Whitney U test, $\mathrm{p}=0.154$ ). The changes in inflammation and deformity grades between initial and followup MRI for both groups are given in Table 4.
The height of the mandibular ramus increased by a median difference of $5.2 \mathrm{~mm}(95 \%$ CI $4.5-5.9 \mathrm{~mm}$, Wilcoxon test, $\mathrm{p}<0.0001)$ from initial to followup MRI, with a median growth rate of $1.4 \mathrm{~mm} /$ year (95\% CI 1.2-1.6 mm). At both MRI studies, the mandibular ramus height was not significantly different between TMJ with and without inflammation or deformation at baseline (Table 5). The growth rates were not significantly different between TMJ with and without inflammation at baseline but tended to be lower in the 
Table 4. Change of inflammation and deformity grades from initial to followup MRI in $76 \mathrm{TMJ}$ of 38 patients with JIA receiving systemic therapy and having TMJ involvement.

\begin{tabular}{lcc}
\hline Grade & $\begin{array}{c}\text { TMJ without } \\
\text { Inflammation } \\
\text { at Baseline }\end{array}$ & $\begin{array}{c}\text { TMJ with } \\
\text { Inflammation } \\
\text { at Baseline }\end{array}$ \\
\hline Grade of inflammation & NA & \\
Improved & $20 / 30(67)$ & $19 / 46(41)$ \\
Stable & $10 / 30(33)$ & $1 / 46(2)$ \\
Deteriorated & & $12 / 46(26)$ \\
Grade of deformity & $2 / 30(7)$ & $31 / 46(67)$ \\
Improved & $23 / 30(77)$ & $3 / 46(7)$ \\
Stable & $5 / 30(17)$ & \\
Deteriorated & & \\
\hline
\end{tabular}

Values are n/N (\%). MRI: magnetic resonance imaging; TMJ: temporomandibular joint; JIA: juvenile idiopathic arthritis; NA: not applicable.

TMJ with inflammation (median difference $-0.2 \mathrm{~mm} / \mathrm{yr}$, Mann-Whitney $\mathrm{U}$ test, $\mathrm{p}=0.273$ ). Overall, the observed growth rates were not significantly different from normal (Figure 1 and Table 5) ${ }^{16,17}$. TMJ with inflammation at baseline tended to have lower growth rates than normal (median difference $-0.15 \mathrm{~mm} / \mathrm{yr}$, Wilcoxon test, $\mathrm{p}=0.140$ ) while TMJ without inflammation at baseline showed no difference (median difference $0.04 \mathrm{~mm} / \mathrm{yr}$, Wilcoxon test, $\mathrm{p}=0.665$ ). The growth rate did not correlate with the grade of inflammation at initial or followup MRI, but showed a weak negative correlation with the degree of deformity at initial $\left(\mathrm{r}_{\mathrm{s}}=-0.257, \mathrm{p}=0.025\right)$ and at followup MRI $\left(\mathrm{r}_{\mathrm{s}}=-0.399, \mathrm{p}<0.001\right)$.

\section{DISCUSSION}

With this longitudinal study we describe the course of TMJ deformity, TMJ inflammation, and clinical findings in 38 children with JIA receiving systemic therapy over a median period of 3.6 years (range 2-8.7 yrs). Reviews have indicated that there are not much data evaluating the efficacy of contemporary systemic treatment on TMJ involvement in patients with JIA ${ }^{18,19}$. While there is anecdotal evidence that systemic therapy may decrease progressive radiographically evident destructive changes of the TMJ and clinically seen facial deformities ${ }^{19}$, only 2 series observed that systemic therapy may be effective in this regard ${ }^{20,21}$. The study by Ince, et al suggested that MTX therapy may minimize TMJ destruction in polyarticular JIA, because 18 patients receiving MTX showed less severe TMJ involvement than 9 patients not receiving $\mathrm{MTX}^{20}$. In a longitudinal study of 84 children with JIA, Twilt, et al showed that the prevalence of patients with condylar alterations decreased from $49 \%$ to $40 \%$ over 5 years when assessed on orthopantomographs, but this improvement was associated with low disease activity and a less extensive therapeutic regimen ${ }^{21}$. Before the widespread use of MTX therapy, Arvidsson, et al observed progression of radiographic condylar and temporal bone abnormalities from $42 \%$ of 60 children with JIA at baseline to $65 \%$ at a 4-year followup examination in the early $1980 \mathrm{~s}^{22}$. We found a slightly higher prevalence of condylar deformity at baseline (22/38 patients, $59 \%)$, which was not significantly different at followup (20/38 patients, 53\%). The overall frequency and degree of TMJ deformation did not change significantly between the MRI studies, but TMJ with arthritis at baseline showed an improvement of the condylar deformity at followup ( $p=0.011)$. In addition, we did not observe any progressive TMJ destruction or intraarticular calcification (deformity grade 4), which in contrast had developed rather frequently (26\% progressive TMJ destruction, $20 \%$ intraarticular calcification) in our previous series of 33 children treated with intraarticular corticosteroid injection ${ }^{10}$. The absence of severe progressive condylar destruction in the current patients taking systemic therapy may explain why the mandibular ramus growth rate remained normal, while it was significantly lower than normal in the aforementioned patients treated with TMJ corticosteroid injection.

The maintained normal mandibular ramus growth and improvement of condylar deformity in TMJ with arthritis at baseline may be the basis for our observation that mandibular skeletal asymmetry tended to decrease in the patients of the current study. This may suggest that systemic treatment could be contributory in preventing craniofacial deformity attributed to impaired mandibular ramus growth resulting from TMJ arthritis in children with JIA. Alternatively, the trend toward lower facial asymmetry could just be a manifestation of normal variation during growth ${ }^{23}$. The current pathophysiological assumption is that disease-modifying antirheumatic

Table 5. Mandibular ramus height and growth rates in 38 patients with JIA receiving systemic therapy and having TMJ involvement.

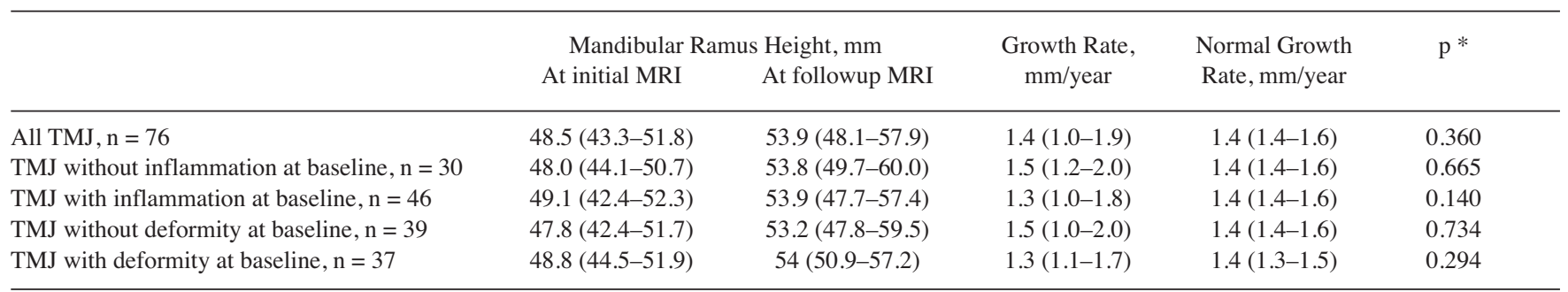

Data are given as median (interquartile range). * Comparison between measured growth rates and age- and sex-matched normal growth rates with Wilcoxon test. MRI: magnetic resonance imaging; TMJ: temporomandibular joint; JIA: juvenile idiopathic arthritis.

Personal non-commercial use only. The Journal of Rheumatology Copyright @ 2020 . All rights reserved 


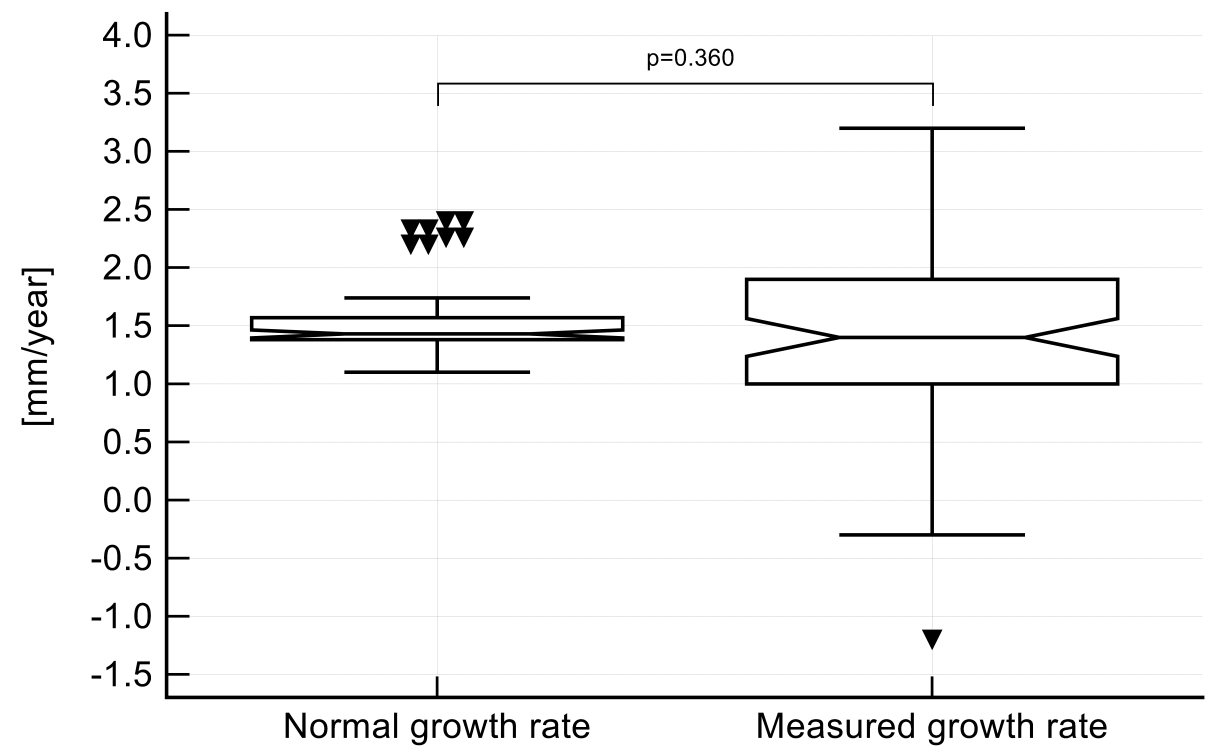

\begin{abstract}
Figure 1. Notched box and whisker plot showing the mandibular ramus growth rate in 38 patients (76 TMJ) during a median followup of 3.6 years (range 2-8.7 yrs, IQR 2.6-4.7 yrs) in comparison to the mean age- and sex-matched normal growth rate $(\mathrm{p}=0.360$, Wilcoxon test $)$. Normal growth rates were calculated from annual increments of cephalographic measurements between condylion and gonion in 102 children from 3 to 16 years of age ${ }^{16,17}$. The central box represents the values from the lower to upper quartile (25th-75th percentile), the middle line represents the median and the whiskers represent the minimum and maximum values with exclusion of outside values (a value that is smaller than the lower quartile minus 1.5 times the IQR or larger than the upper quartile plus 1.5 times the IQR). TMJ: temporomandibular joint; IQR: interquartile range.
\end{abstract}

drugs reduce inflammation in the TMJ and therefore allow for normal development of the condyle and growth of the mandibular ramus at the condylar growth zone. This hypothesis is supported by our cohort in 3 ways. First, we found significantly less inflammation in TMJ at followup than at baseline. Second, mandibular ramus growth rate was negatively correlated with condylar deformity. Third, the mandibular ramus growth rate was normal, although it tended to be lower in TMJ with than without inflammation at baseline. To our surprise, there was no correlation between the degree of inflammation seen on MRI and the growth of the mandibular ramus. This may indicate that TMJ arthritis was sufficiently controlled in our patients. However, this finding may also indicate that growth is not so much influenced by the inflammation per se, but growth impairment is the result of the structural damage to the growth zone of the mandibular condyle, which again is supported by the observed negative correlation of the growth rate with condylar deformity. The fact that mandibular ramus growth may be normal despite the presence of low-grade inflammation on MRI could be used as an argument against treating TMJ arthritis aggressively with intraarticular corticosteroids. Another argument against intraarticular corticosteroids would be that we want to avoid the risk of creating steroid-induced severe TMJ deformities because that limits mandibular growth.

The clinical findings in this cohort confirm that orofacial examination has a poor diagnostic value for predicting the presence of TMJ arthritis ${ }^{14,24}$. Pain was present in only $13 \%$ and $9 \%$ of TMJ at initial and followup examination and did not correlate with MRI signs of inflammation or deformity. Overall, MOC was normal at initial and followup examination, and did not correlate with the MRI findings. Facial asymmetry was not significantly different between examinations but tended to improve at followup ( $\mathrm{p}=0.056)$.

The main limitation of our study is its retrospective and uncontrolled design with variable medication and imaging intervals. From our results, it is not possible to draw any conclusions on the efficacy of different medications or combinations thereof. By examining the patients at only 2 timepoints, we cannot account for possible effects of TMJ arthritis fluctuation on the observed changes. The study cohort reflects our current practice of treating JIA with systemic medication in cases with severe TMJ inflammation or when local treatment of other joints is not sufficient to control disease activity. MRI of the TMJ was usually conducted when the presence of TMJ inflammation would have changed the treatment approach or for assessing treatment response of confirmed TMJ arthritis. By measuring only the height of the mandibular ramus, we did not account for all components of vertical mandibular growth. Evaluation of appositional growth, changes in the gonion area, antegonial notching, and backward-rotation of the mandibular corpus would require cephalography or 3-D

Personal non-commercial use only. The Journal of Rheumatology Copyright ( $) 2020$. All rights reserved. 
computed tomography, which was not available in our patients. Nonetheless, by showing normal growth of the mandibular ramus height we assume normal condylar growth that is not impaired by TMJ arthritis. The use of historical cephalographic data for normal mandibular ramus growth is another limitation to our growth assessment. To our knowledge, this is the only available data in the literature that allows calculation of normal mandibular ramus growth rate. Because the growth rates in both our groups of TMJ with and without arthritis at baseline did not differ from the historical normal growth rates, they may still be accurate.

Another limitation is the measurement error of our assessment methods, which may not allow measuring shortterm mandibular ramus growth and change of MRI findings in the TMJ accurately in a single patient. According to Markic, et $a l^{9}$, MRI measurements of the mandibular ramus height can be performed with a mean difference of $0.2 \mathrm{~mm}$ (95\% limits of agreement -2.4 to $2.9 \mathrm{~mm}$ ). With a mean normal growth rate of $1.6 \mathrm{~mm} / \mathrm{year}$ (range $0.7-2.6$ $\mathrm{mm} / \mathrm{yr})^{16,17}$, short-term growth rates over 3 months may show a mean measurement error of up to $\pm 50 \%$, whereas for longterm growth rates over 5 years, the mean measurement error is estimated at $\pm 5 \%$. The grading of the TMJ inflammation and deformity was performed by 2 readers in consensus, to improve reliability of our assessment. The reliability of the applied grading system has been tested elsewhere ${ }^{25}$, showing high reliability both for the inflammatory and deformity domain (average-measure ICC 0.92 and 0.96) and excellent smallest detectable differences (29\% and 23\%). While these measurement errors may be substantial when assessing a single TMJ, they should be canceled out when comparing mean growth rates and MRI scores of groups of joints as done in the current work.

Further studies are needed to support the portrayed findings, and should especially focus on evaluating the efficacy of different systemic medications, and address the longterm effect on TMJ morphology and overall craniofacial development.

With this retrospective longitudinal study we suggest that systemic treatment of TMJ arthritis in children with JIA may reduce inflammatory changes seen on MRI, preserves osseous TMJ morphology, and maintains normal mandibular ramus growth over a period of at least 2 years. This is clearly in contrast to an earlier cohort treated with corticosteroid TMJ injections, in which TMJ deformity deteriorated and mandibular ramus growth was impaired. Our findings are also in stark contrast to the body of literature from the pretherapeutic era of JIA, at least regarding growth and damage.

\section{REFERENCES}

1. Larheim TA, Doria AS, Kirkhus E, Parra DA, Kellenberger CJ, Arvidsson LZ. TMJ imaging in JIA patients - an overview. Semin Orthod 2015;21:102-10.
2. Cannizzaro E, Schroeder S, Muller LM, Kellenberger CJ, Saurenmann RK. Temporomandibular joint involvement in children with juvenile idiopathic arthritis. J Rheumatol 2011;38:510-5.

3. Arvidsson LZ, Fjeld MG, Smith HJ, Flato B, Ogaard B, Larheim TA. Craniofacial growth disturbance is related to temporomandibular joint abnormality in patients with juvenile idiopathic arthritis, but normal facial profile was also found at the 27-year follow-up. Scand J Rheumatol 2010;39:373-9.

4. Weiss PF, Arabshahi B, Johnson A, Bilaniuk LT, Zarnow D, Cahill AM, et al. High prevalence of temporomandibular joint arthritis at disease onset in children with juvenile idiopathic arthritis, as detected by magnetic resonance imaging but not by ultrasound. Arthritis Rheum 2008;58:1189-96.

5. Muller L, Kellenberger CJ, Cannizzaro E, Ettlin D, Schraner T, Bolt IB, et al. Early diagnosis of temporomandibular joint involvement in juvenile idiopathic arthritis: a pilot study comparing clinical examination and ultrasound to magnetic resonance imaging. Rheumatology 2009;48:680-5.

6. Kuseler A, Pedersen TK, Herlin T, Gelineck J. Contrast enhanced magnetic resonance imaging as a method to diagnose early inflammatory changes in the temporomandibular joint in children with juvenile chronic arthritis. J Rheumatol 1998;25:1406-12.

7. Kellenberger CJ, Arvidsson LZ, Larheim TA. Magnetic resonance imaging of temporomandibular joints in juvenile idiopathic arthritis. Semin Orthod 2015;21:111-20.

8. Kellenberger CJ, Junhasavasdikul T, Tolend M, Doria AS. Temporomandibular joint atlas for detection and grading of juvenile idiopathic arthritis involvement by magnetic resonance imaging. Pediatr Radiol 2018;48:411-26.

9. Markic G, Muller L, Patcas R, Roos M, Lochbuhler N, Peltomaki T, et al. Assessing the length of the mandibular ramus and the condylar process: a comparison of OPG, CBCT, CT, MRI, and lateral cephalometric measurements. Eur J Orthod 2015;37:13-21.

10. Lochbuhler N, Saurenmann RK, Muller L, Kellenberger CJ. Magnetic resonance imaging assessment of temporomandibular joint involvement and mandibular growth following corticosteroid injection in juvenile idiopathic arthritis. J Rheumatol 2015; 42:1514-22.

11. Petty RE, Southwood TR, Manners P, Baum J, Glass DN, Goldenberg J, et al. International League of Associations for Rheumatology classification of juvenile idiopathic arthritis: Second revision, Edmonton, 2001. J Rheumatol 2004;31:390-2.

12. Hauser R, Schroeder S, Cannizzaro E, Muller L, Kellenberger CJ, Saurenmann RK. How important is early magnetic resonance imaging of the temporomandibular joint for the treatment of children with juvenile idiopathic arthritis: a retrospective analysis. Pediatr Rheumatol 2014;12:36.

13. Stoustrup P, Resnick CM, Pedersen TK, Abramowicz S, Michelotti A, Kuseler A, et al. Standardizing terminology and assessment for orofacial conditions in juvenile idiopathic arthritis: international, multidisciplinary consensus-based recommendations. J Rheumatol 2019;46:518-22.

14. Keller H, Muller LM, Markic G, Schraner T, Kellenberger CJ, Saurenmann RK. Is early TMJ involvement in children with juvenile idiopathic arthritis clinically detectable? Clinical examination of the TMJ in comparison with contrast enhanced MRI in patients with juvenile idiopathic arthritis. Pediatr Rheumatol Online J 2015;13:56.

15. Muller L, van Waes H, Langerweger C, Molinari L, Saurenmann RK. Maximal mouth opening capacity: Percentiles for healthy children 4-17 years of age. Pediatr Rheumatol Online J 2013;11:17

16. Tracy WE, Savara BS. Norms of size and annual increments of five anatomical measures of the mandible in girls from 3 to 16 years of age. Arch Oral Biol 1966;11:587-98.

17. Savara BS, Tracy WE. Norms of size and annual increments for five

Personal non-commercial use only. The Journal of Rheumatology Copyright @ 2020 . All rights reserved. 
anatomical measures of the mandible in boys from three to sixteen years of age. Arch Oral Biol 1967;12:469-86.

18. Te Veldhuis EC, Te Veldhuis AH, Koudstaal MJ. Treatment management of children with juvenile idiopathic arthritis with temporomandibular joint involvement: a systematic review. Oral Surg Oral Med Oral Pathol Oral Radiol 2014;117:581-9.

19. Stoll ML, Kau CH, Waite PD, Cron RQ. Temporomandibular joint arthritis in juvenile idiopathic arthritis, now what? Pediatr Rheumatol Online J 2018;16:32.

20. Ince DO, Ince A, Moore TL. Effect of methotrexate on the temporomandibular joint and facial morphology in juvenile rheumatoid arthritis patients. Am J Orthod Dentofacial Orthop 2000;118:75-83.

21. Twilt M, Schulten AJ, Verschure F, Wisse L, Prahl-Andersen B, van Suijlekom-Smit LW. Long-term followup of temporomandibular joint involvement in juvenile idiopathic arthritis. Arthritis Rheum 2008;59:546-52.
22. Arvidsson LZ, Flato B, Larheim TA. Radiographic TMJ abnormalities in patients with juvenile idiopathic arthritis followed for 27 years. Oral Surg Oral Med Oral Pathol Oral Radiol Endod 2009;108:114-23.

23. Liukkonen M, Sillanmaki L, Peltomaki T. Mandibular asymmetry in healthy children. Acta Odontol Scand 2005;63:168-72.

24. Stoustrup P, Twilt M, Spiegel L, Kristensen KD, Koos B, Pedersen $\mathrm{TK}$, et al. Clinical orofacial examination in juvenile idiopathic arthritis: international consensus-based recommendations for monitoring patients in clinical practice and research studies. J Rheumatol 2017;44:326-33.

25. Tolend MA, Twilt M, Cron RQ, Tzaribachev N, Guleria S, von Kalle T, et al. Toward establishing a standardized magnetic resonance imaging scoring system for temporomandibular joints in juvenile idiopathic arthritis. Arthritis Care Res 2018;70:758-67. 